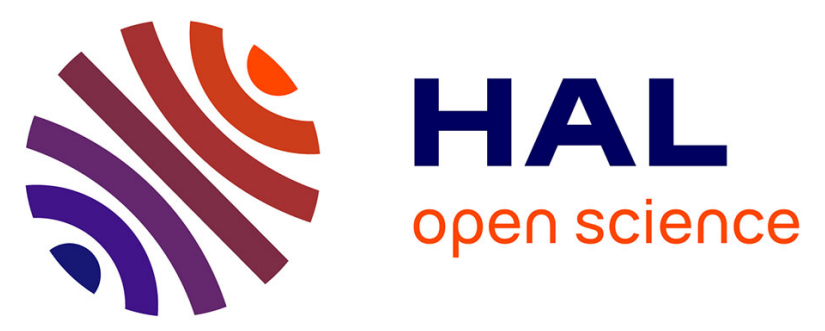

\title{
Fabrication of flexible transparent Ag square-shaped mesh electrode by top-flat nanosecond laser ablation
}

Dongfeng Qi, Xiaohan Yu, Qingwei Zhang, Dongwoo Paeng, Xiang Han, Andong Wang, Donglin Huang, Letian Wang, Zifeng Zhang, Songyan Chen, et al.

\section{To cite this version:}

Dongfeng Qi, Xiaohan Yu, Qingwei Zhang, Dongwoo Paeng, Xiang Han, et al.. Fabrication of flexible transparent Ag square-shaped mesh electrode by top-flat nanosecond laser ablation. Optics Letters, 2020, 45 (4), pp.901. 10.1364/OL.384463 . hal-03160522

\section{HAL Id: hal-03160522 \\ https://hal.science/hal-03160522}

Submitted on 10 Mar 2021

HAL is a multi-disciplinary open access archive for the deposit and dissemination of scientific research documents, whether they are published or not. The documents may come from teaching and research institutions in France or abroad, or from public or private research centers.
L'archive ouverte pluridisciplinaire HAL, est destinée au dépôt et à la diffusion de documents scientifiques de niveau recherche, publiés ou non, émanant des établissements d'enseignement et de recherche français ou étrangers, des laboratoires publics ou privés. 


\title{
Fabrication of flexible transparent Ag square- shaped mesh electrode by top-flat nanosecond laser ablation
}

\author{
Dongfeng QI, 1,2,3,7 Xiaohan Yu, ${ }^{1,2}$ QIngwei Zhang, ${ }^{1,2}$ Dongwoo Paeng, ${ }^{3}$ \\ Xiang Han, ${ }^{4}$ Andong Wang, ${ }^{5}$ Donglin Huang, ${ }^{4}$ Letian Wang, ${ }^{3}$ Zifeng \\ Zhang, ${ }^{6}$ SongYan Chen, ${ }^{1,2}$ Shixun Dal, ${ }^{1,2}$ And XIANg Shen, $, 1,2,8$ \\ ${ }^{1}$ Laboratory of Infrared Materials and Devices, Ningbo University, Ningbo, Zhejiang province, 315211, People's Republic of China \\ ${ }^{2}$ Key Laboratory of Photoelectric Materials and Devices of Zhejiang Province, Ningbo University, Ningbo, Zhejiang province, 315211, People's \\ Republic of China \\ ${ }^{3}$ Laser Thermal Laboratory, Department of Mechanical Engineering, University of California, Berkeley, Berkeley, CA 94720-1740, USA \\ ${ }^{4}$ Department of Physics, Xiamen University, Xiamen, Fujian province 361005, People's Republic of China \\ ${ }^{5}$ Aix-Marseille University, CNRS, LP3 UMR 7341, 13009 Marseille, France \\ ${ }^{6}$ College of Mechanical and Electronic Engineering, Chaohu University, Hefei, Anhui 230000, People's Republic of China \\ 7e-mail:qidongfeng@nbu.edu.cn \\ 8e-mail:shenxiang@nbu.edu.cn
}

Received XX Month XXXX; revised XX Month, XXXX; accepted XX Month XXXX; posted XX Month XXXX (Doc. ID XXXXX); published XX Month XXXX

We report a facile top-flat square nanosecond (ns) laser direct writing (LDW) ablation technique in a thin silver film substrate to fabricate square silver honeycomb structure of flexible transparent electrodes. The square silver honeycomb structures feature smooth surface morphology, excellent edge definition, mechanical stability, strong adhesion to the substrate, and favorable resistance and transparency. Due to the facile cold machining by one-step top-flat square nanosecond LDW ablation of $\mathrm{Ag}$ film, different thicknesses of $\mathrm{Ag}$ mesh electrodes $(20 \mathrm{~nm}, 50 \mathrm{~nm}, 160 \mathrm{~nm})$ of glossy metallic honeycomb surfaces with smooth morphology and excellent edge definition can be fabricated. In particular, this strategy enables fabrication of a high square honeycomb areal density (ablated square honeycomb to the total area) $\mathrm{Ag}$ mesh, substantially improving transparency $(>85 \%)$ without considerably sacrificing conductivity $(<23.2 \Omega \quad$ sq-1 unit of resistance). Consequently, the proposed metallic honeycomb structure shows compatibility with polyethylene naphthalate (PEN) flexible substrate for silver-based wearable electronic devices without any protective layer over the electrodes.

http://dx.doi.org/10.1364/OL.99.099999

Flexible transparent conductive electrodes (FTCEs) are essential components of various electronic and optoelectronic devices, such as touch screen panels (TSPs), organic light-emitting diodes (OLEDs) and solar cells. For such transparent conductive devices, indium tin oxide (ITO) prevails in the market of transparent conducting materials.[1-5] However, due to the increasing demand for flexible and wearable electronic devices, ITO suffers from several intrinsic drawbacks, since its brittleness causes cracks upon flexible test and high material cost due to indium is the rare earth material [6-9]. Researchers have begun to seek new flexible electrode materials such as carbon nanotubes, graphene, metal (silver, copper) nanowires and transparent metal grids [10-12]. Carbon nanotubes and graphene have good flexibility can sustain tension of $100 \%$ stress stretching, but as electrode materials, the conductivity and transmittance properties of carbon nanotubes or graphite are much smaller than those of ITO materials [13]. The conductivity and transmittance of metal silver and copper nanowires are 1 2 orders of magnitude higher than that of carbon nanotubes, and especially for $\mathrm{Ag}$ nanowires, the photoelectric properties (resistance: $20 \Omega \mathrm{sq}^{-1}$, transmittance: $90 \%$ ) are comparable to those of ITO materials $[14,15]$. However, the random $\mathrm{Ag}$, $\mathrm{Cu}$ and $\mathrm{Cu}$ (core)-Ni (shell) nanowires network fabrication using mold, bulk heating, annealing process or fast plasmonic nano-welding method increases material waste, edge roughness and processing time $[13,16]$. In addition, the performance of nanowire-based transparent electrodes largely depends on the property of nanowire such as crystallization and aspect ratio. Some methods to elongate the length of the silver nanowire and improve the crystallization of nanowires have been reported recently, such as nano-welding to enhance contact of individual nanowires and pressurization at high temperature [10,17]. However, it makes the whole process more complicated and timeconsuming.

Laser direct writing (LDW) process can effectively fabricate grid structures in the surface of solution-processed nanoparticles (NPs) thin films $[1,2,14,18]$. Compared with traditional processing methods, LDW process of NPs of different pitches has unique advantage for the preparation of flexible transparent electrodes. Since metal films thinner than the light absorption depth is still conductive, and metal grid fabrication methods via NPs sintering and reductive sintering have been demonstrated for transparent electrodes. In this case, utilizing metal films compatible with LDW processes is a new route for the fabrication of flexible transparent electrodes. The laser direct sintering and reduction process of solution-processed NPs, in particular, can be applied to fabricate flexible transparent devices owing to lowered thermal stress exerting on thermally vulnerable 
polymer substrates.[1] Recently, NPs or NPs composed of Ag, $\mathrm{Au}, \mathrm{Cu}$ and $\mathrm{Ni}$ have been widely employed because these nano-materials can be easily maintained to grid electrodes of different pitches simply by sintering nanoparticles or reductive sintering metal oxide $[1,2,19]$. Through localized laser reductive sintering and sintering of metal oxide or metal NPs, highly conductive metal electrodes can be successfully fabricated. However, resolution of electrodes fabricated through these methods is limited due to the large and uneven size of the NPs and metal oxide [20]. Besides, copper and nickel can be susceptible to re-oxidation at the laser direct writing process, which causes degradation of its electrical properties [20-23]. In this case, another route would be utilizing deposition-processed metal films compatible with laser direct ablation process to fabricate transparent electrode [15, 23]. Metal patterns on flexible substrates not only meet the high transmittance, large area and low cost performance for flexible transparent electrode, but also retain the low conductivity and the ductility of the metal film [24, 25]. Besides, the LDW ablation process of ultrathin deposition-processed metal films enabled material removal without inflicting thermal damage on the underlying flexible polymer substrates [24]. As a result, metallic honeycomb arrays can be directly produced on various heat-sensitive flexible substrates. However, properties and morphologies of the electrodes are not uniform after Gaussian pulse irradiation [23, 24]. For the Gaussian beam, the temperature gradient in the beam spot results in different thermodynamic processes in the center of the spot and the peripheral spot, which affects the quality of the metallic honeycomb transparent electrodes [1, 2, 24]. Secondly, in the process of fabrication of metallic honeycomb electrode through pulsed laser ablation metal thin films, laser fluence at the peripheral of Gaussian spot is between the melting threshold and the ablation threshold of the material. In this case, the temperature gradient drives the molten materials to the outer edge of the spot, and the cylindrical ring or droplet finger structures are formed in the peripheral dewetting zone due to the solidification of transported matter following the laser pulse exposure. (the height is more than twice times thicker than that of the original film) $[2,22,23]$. And for oxygen sensitive metal films, such as $\mathrm{Cu}, \mathrm{Ni}$ and other materials, Gaussian pulse direct ablation process not only causes uneven metallic honeycomb, but the re-oxidized metallic materials can reduce the conductive properties of metallic honeycomb electrodes [20-23]. In summary, the roughness of the metallic electrode reduces the light transmittance, and the cylindrical ring, droplet finger structures and the inhomogeneous of the compound metallic electrode increase the leakage of the electronic device, which largely restricts the development of laser direct ablation process for the fabrication of high quality flexible transparent electrode.

In this work, Ag honeycomb FTCEs were produced by a facile cold machining after one-step top-flat square nanosecond LDW ablation of Ag film under ambient conditions. Here, the term "cold machining" refers to the phenomenon wherein ablation occurs just at the square spot without outward radial mass transfer of molten metal, resulting in no structural deformation and damage on the edge of metallic honeycombs. Besides, square metallic honeycombs can effectively improve the areal density according to the circle metallic honeycombs, and the higher areal density results in the higher transparency. In this condition, compare with the Gaussian beam LDW ablation process, the top-flat square LDW ablation process not only maintain Ag mesh with higher electrical conductivity but also higher transparency. The electrical performance of Ag metallic honeycombs FTCEs on polyethylene naphthalate (PEN) substrate is maintained even after bending, folding, twisting, squeezing and ultrasonic bath tests. In addition, the transmittance and conductivity of the Ag metallic honeycombs electrode enable the fabrication of a transparent touch screen panel.
The LDW ablation process was applied on the as-prepared thin silver film using a nanosecond laser ( $\mathrm{Nd}: \mathrm{YVO}_{4}$, wavelength:532 nm, duration time: $22.5 \mathrm{~ns}$ ). A schematic of the laser setup for the fabrication of Ag mesh FTCEs is shown in Fig. 1(a). Upon irradiation of nanosecond laser pulses, a top-flat square beam can be modulated by a diffractive optical element, resulting in the fabrication of square honeycomb arrays in silver film on a polyethylene naphthalate (PEN) substrate. In this case, the Ag mesh not only has the superiority of the higher transparency but also maintains the good electronic conductivity of silver.

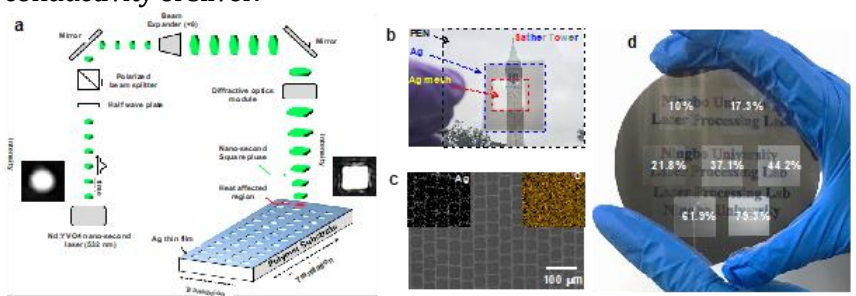

Fig. 1. (a) Fabrication schematics of Ag mesh FTCEs. (b) Photograph of a transparent conductor on a PEN substrate (PEN substrate in the black-boxed region, Ag film in the blue-boxed region and high-density Ag mesh in the red-boxed region), and the background is the Sather Tower of UC Berkeley. (c) Top-view SEM image of Ag mesh-type electrodes on the PEN substrate. The insets show elemental mapping images for Ag and C of the electrodes. (d) Photo-image of several $2 \mathrm{~cm}$ $\times 2 \mathrm{~cm}$ mesh patterns on the 4 -inch quartz substrate, and the number indicates the corresponding honeycomb density of the square mesh patterns.

Fig. 1(b) shows a $5 \mathrm{~cm} \times 5 \mathrm{~cm}$ flexible transparent conductive electrode on PEN composed of mesh-type $\mathrm{Ag}$ with a square honeycomb density of $79 \%$ in the red box ( $2 \mathrm{~cm} \times 2 \mathrm{~cm}$ area); no laser ablation of the Ag film on PEN is observed in the blue box. It is noted that this demonstration uses just a $1 \mathrm{kHz}$ laser, and the total laser processing time required for scanning a $2 \mathrm{~cm} \times 2 \mathrm{~cm}$ area with a scanning speed of $25 \mathrm{~mm} / \mathrm{s}$ is only approximately $10 \mathrm{~min}$, which can be possibly shortened by further optimization, such as higher laser repetition and higher scanning speed. A $5 \mathrm{~cm} \times 5 \mathrm{~cm} \mathrm{Ag}$ mesh electrode could be fabricated within 2-5 s using an array of $30 \mu \mathrm{m}$ square honeycomb, a laser at $1 \mathrm{MHz}$ pulse repetition, and $10 \mathrm{~m} / \mathrm{s}$ sample translation speed to write the square honeycomb patterns. In addition, Fig. 1(c) shows the SEM of the patterned Ag FTCE by taking advantage of high-resolution top-flat square LDW ablation processing. Interestingly, due to the uniform of the laser intensity in the top-flat square spot, the measured size of each honeycomb is approximately $30 \mu \mathrm{m}$, which is similar with the size of the beam spot, and no structural deformation and damage on the edge of honeycombs that are detrimental to the optical characteristics are observed. The uniformity of $\mathrm{Ag}$ mesh upon the top-flat square LDW ablation processing is confirmed by the elemental mapping images of $\mathrm{Ag}$ and $\mathrm{C}$, as shown in the insets of Fig. 1(c). It is worth noting that the Celements detected in the background are partly from the flexible PEN substrate. The two images show a clear contrast between Ag and C elements in the electrodes, suggesting that the top-flat square LDW laser irradiation can cleanly removes the irradiated parts by cold machining technology. Because of heat diffusion on the Ag film induced by the laser irradiation, and the $\mathrm{Ag}$ ratio at the rim is higher than that at the edge of the honeycomb, which may lead to a reduced conductivity of the electrodes. Some references also mentioned that the smooth and flat metal electrode could improve characteristics of the transparent electrode, such as transparency, conductivity and stability.[1,2,24] Fig. 1(d) shows the effect of the square honeycomb arrays on substratebased transmittance of the Ag mesh electrodes on a 4-inch quartz substrate. By optimizing the density and size of the square honeycomb 
patterns, we obtained patterned electrodes of high transmittance while maintaining low sheet resistance. The numbers on each pattern ( $2 \mathrm{~cm} \times 2 \mathrm{~cm}$ Ag mesh pattern) in Fig. 1(d) indicate the density of the square honeycombs in the Ag film. The picture was taken on printed letters as a background to illustrate the electrode transmittance.
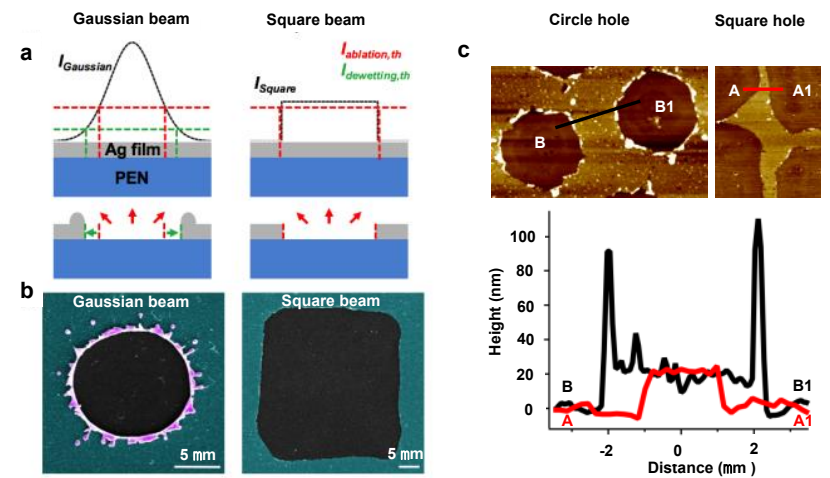

Fig. 2. (a) Schematics of morphology transformations of Ag film upon Gaussian LDW ablation processing and ultrathin Ag film upon top-flat square LDW ablation processing. I I Gaussian and $I_{\text {square }}$ are the laser intensity profiles for the Gaussian laser beam and the top-flat square laser beam, respectively. The intensity profile is presented with a dashed line. The solid arrows indicate the transport directions of molten silver upon the laser irradiation. (b) The typical SEM images of the corresponding $20 \mathrm{~nm}$ silver film on PEN substrate after Gaussian beam and Top-flat square LDW ablation processes, and fluences of the irradiation laser $1.44 \mathrm{~J} / \mathrm{cm}^{2}$ for Gaussian beam, and $490 \mathrm{~mJ} / \mathrm{cm}^{2}$ for top-flat square beam, and the scale bars are $5 \mu \mathrm{m}$. (c) AFM image of the Ag FTCEs.

Furthermore, the atomic force microscopy (AFM) image shows that the height and morphology between the film and the ablated spot after Gaussian and top-flat square beam irradiation, as shown in Fig. 2(c). For the Gaussian beam LDW ablation processing, the nominal thickness of the electrode measured by AFM is approximately $25 \mathrm{~nm}$. Condensed and re-solidified Ag clusters are found near the peripheries of the honeycombs due to the stronger melt expulsion and ablation. In addition, the rim of the ablated honeycomb is around two times thicker than the original film, suggesting outward radial mass transfer of molten metal, as shown in Fig. 2(a). For the top-flat square LDW ablation processing, the rim droplet fingers will not appear in the edge of the spot, and the inner honeycomb is smooth and neat, as shown in Fig. 2(c). It is worth noting that the edges of the top-flat square LDW ablation electrode mesh are sharp, suggesting that this top-flat square LDW ablation process can be applied to a high-resolution electrode fabrication, as shown in Fig. 2(a). In this case, upon the top-flat square ns LDW ablation, square silver honeycomb arrays can be produced in a silver film on a flexible polymer substrate, resulting in higher transparency while maintaining the good electrical conductivity of silver. Besides the advantage of the top-flat LDW ablation processing for the fabrication of $\mathrm{Ag}$ mesh electrode, square honeycomb arrays can effectively improve the areal densities according to the circle honeycomb arrays (from $52 \%$ to $79 \%$ ). The areal density is herein defined as the ratio of the honeycomb area after ablation to the total area. By changing the scanning speed, honeycomb arrays with different areal densities can be maintained. For the square arrays, the areal density can be fabricated as high as $79 \%$. However, the areal density of circle arrays is only $52 \%$. The higher areal density result in the higher transparency, in this condition, compare with the Gaussian beam LDW processing, the top-flat square LDW ablation processing can not only maintain Ag mesh with higher electrical conductivity but also higher transparency, which can be defined as cold machining technology.
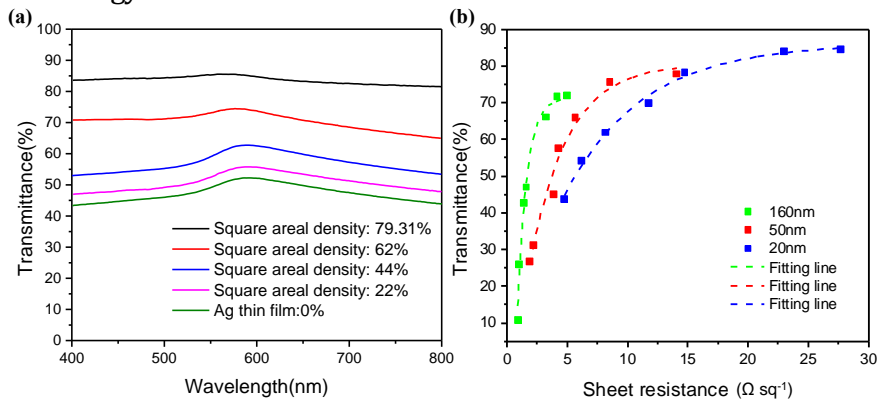

Fig. 3. (a) The transmittance spectra of Ag FTCEs with different areal densities (20 nm thicknesses). (b) Plot of sheet resistance versus transmittance at $550 \mathrm{~nm}$ wavelength of Ag FTCEs at different square honeycomb areal densities and different thickness of Ag film.

The transmittance of the Ag FTCEs can be controlled by synchronizing the speed of the stage. As-prepared Ag film (square honeycomb areal density $=0$ ) yields transmittance of $46 \%$ at the wavelength of $550 \mathrm{~nm}$, shown in Fig. 3(a), while the maximum transmittance of $85.1 \%$ was achieved when the square honeycomb areal density is 0.79 . Fig. 3(b) displays a plot of transmittance at 550 $\mathrm{nm}$ versus sheet resistance. The balance between transmittance and sheet resistance can be adjusted by altering the areal density and thickness of the mesh patterns. Due to the smaller line-width and higher square honeycomb density, the $160 \mathrm{~nm} \mathrm{Ag}$ FTCEs demonstrate high optical transmittance (70.8\% at $550 \mathrm{~nm}$ wavelength) with very low sheet resistance $\left(5 \Omega \mathrm{sq}^{-1}\right)$, as shown in Fig. 3(b). These successful fabrications confirm that the square honeycomb arrays and these key fabrication steps can be reliably scaled down to a sub-micrometer range. Decreasing the thickness of the Ag FTCEs while maintaining same line-width can also effectively improve the transmittance (up to 85.1\%). Fig. 3(a) displays the spectra and sheet resistance of a highly transparent Ag FTCEs on a $4 \times 4 \mathrm{~cm}$ large PEN substrate with different square honeycomb areal density and Ag-mesh thickness of 20,50 and $160 \mathrm{~nm}$, respectively. Moreover, the suggested top-flat square LDW ablation process method in this work can fabricate electrodes invisible to naked eyes. Because of large line spacing and thin thickness, the $\mathrm{Ag}$ FTCE (20 nm) exhibits high optical transmittance (85.1\%) and retains low sheet resistance $\left(22.5 \Omega \mathrm{sq}^{-1}\right)$. An extremely low sheet resistance of $5 \Omega \mathrm{sq}^{-1}$ was observed for the $160 \mathrm{~nm}$ thick Ag FTCEs, and the transmittance at the $550 \mathrm{~nm}$ wavelength was still above $70 \%$. In similar fashion, various combinations of optical transparency and sheet resistance can be obtained for various applications by optimizing the geometric parameters of the $\mathrm{Ag}$ mesh. To gain further insight into how Ag film thickness affects the overall performance of the Ag FTCEs, a commonly used figure of merit (FoM), namely the ratio of electrical conductance to optical conductance, was calculated for all the FTCEs displayed in Fig. 3(b) by using the following widely accepted expression $[26,27]$

$$
T_{\lambda}=\left(1+\frac{188.5}{R_{S}} \frac{\sigma_{O P}(\lambda)}{\sigma_{D C}}\right)^{-2}
$$

Where $\sigma_{O P}$ and $\sigma_{D C}$ are optical conductivity and electrical conductivity of thin metal electrode, respectively. The presented data indicate that the metal thickness has a considerable effect on the sheet resistance and hence on the value of the FoM $\left(\sigma_{D C} / \sigma_{O P}\right)$ by increasing the electrical conductivity of a thicker metal mesh without substantially sacrificing transmittance.

As shown in Fig. 4(a), the transparent Ag mesh on PEN was used as a conductor in a circuit to connect a light-emitting diode (LED) under the voltage of $3 \mathrm{~V}$. There was no noticeable brightness change of the 
LED under folding, twisting and squeezing of the electrode. To identify the long-term mechanical reliability of the Ag mesh electrode, a cyclic bending test was performed. Fig. 4(b) shows the Ag mesh on a PEN substrate test of mechanical stability under cyclic tensile and compressive bending stress. A $2 \mathrm{~cm} \times 5 \mathrm{~cm} \mathrm{Ag} \mathrm{mesh} \mathrm{pad} \mathrm{was}$ fabricated on the PEN substrate. Bending of the pad is created by moving one end at $60 \mathrm{~mm} / \mathrm{s}$ of motion speed and a frequency of $1 \mathrm{~Hz}$, yielding a radius of curvature of $r=7 \mathrm{~mm}$ at the maximum bending deformation. The resistance of the electrode was measured at the flat state after several bending cycles. After over 10000 cycles, for all three different silver films (20 nm, $50 \mathrm{~nm}, 160 \mathrm{~nm})$, the measured resistance variation $\left(\Delta R / R_{0}\right)$ is less than $3 \%$. Moreover, From the SEM images in Fig. 4(b), for all three different thicknesses Ag film, the Ag mesh did not detach from the substrate, and morphology of the Ag mesh did not change much after 10000 cycles bending test.. Since laser irradiation imparts a highly localized temperature field, it is a suitable tool for processing heat-sensitive flexible polymer substrates, and morphological structures of the Ag mesh remained unchanged. This superior bendability is attributed to the strong adhesion of the Ag film (sputtering technology) to the PEN substrate and also to the smooth of Ag square honeycomb arrays. The ultrasonic bath tests performed with $\mathrm{Ag}$ mesh electrode further confirmed strong adhesion of the electrodes on the PEN substrate even though the line-width is only 1 $\mu \mathrm{m}$.
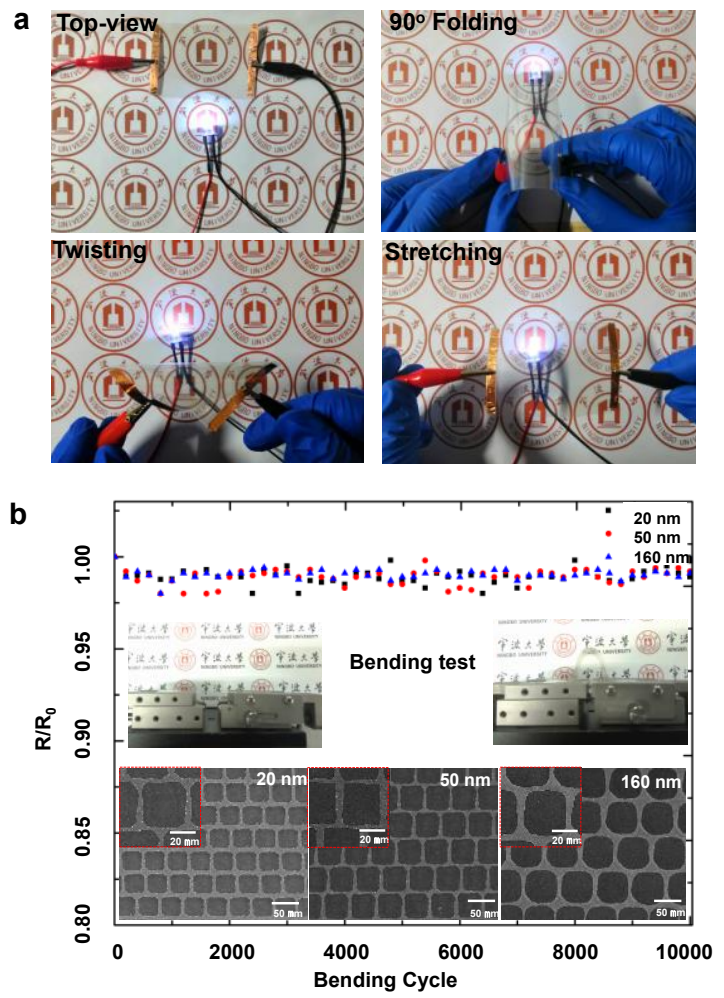

Fig. 4. (a) Mechanical durability test with folding, twisting and squeezing with bare hands. Photographs of Ag mesh based FTCE, and lighting of an LED with an external power supply (3V). (b) The cyclic bending test up to 10000 cycles to show electrical and mechanical stability, and the corresponding SEM images showing morphology in the Ag mesh on PEN substrate after repeated tensile bending 10000 cycles) to radius of $7 \mathrm{~mm}$, the honeycomb areal densities for three thickness Ag mesh are all around 70\%.
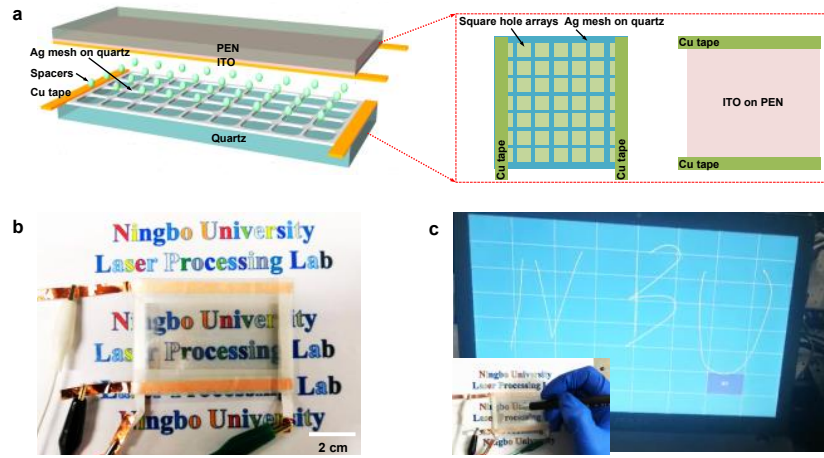

Fig. 5. (a) Schematic diagram of the touch screen panel using Ag mesh on a quartz substrate. (b) Photographs of the four-wire resistive touch screen panel at flat. (c) A touch panel demonstration with the Ag honeycomb on quartz film. Touch screen panel (TSP) attached on a LCD monitor and the schematics of a TSP. Demonstration of the TSP operation fabricated by Ag mesh and ITO-PEN film. "NBU" was written on the TSP.

As a device demonstration of the Ag mesh electrode, a flexible transparent four-wire resistive touch screen panel was fabricated, as shown in Fig. 5 . The $5 \mathrm{~cm} \times 7 \mathrm{~cm}$ Ag mesh-type with square density of $79 \%$ on the quartz substrate (Rs: $23.2 \Omega \mathrm{sq}^{-1}, \mathrm{~T}: 85.1 \%$ at $550 \mathrm{~nm}$ ) were fabricated as shown in Fig. 5(a). No protective coating layer such as PEDOT:PSS was required for the transparent Ag mesh electrode due to strong adhesion on the PEN or quartz substrate. An ITO-PET film (Rs: $100 \Omega \mathrm{sq}^{-1}, \mathrm{~T}$ : $86 \%$ at $550 \mathrm{~nm}$, Teonex) was used as a counter electrode. Two slips of copper tape were attached to the top and bottom sides of the Ag mesh while another two slips were attached to the left and right sides of the ITO-PET film. Due to the transparency of the Ag mesh electrodes, the colored letters beneath the Touch screen panel (TSP) are visible, as shown in Fig. 5(b). The functionality of the touch screen panel is confirmed by writing the University name was evaluated as displayed in Fig. 5(c).

In summary, flexible-compatible maskless Ag honeycomb electrode was prepared by selective LDW ablation of silver thin films deposited via magnetron sputtering system. Thin $(20 \mathrm{~nm}, 50$ $\mathrm{nm}$ and $160 \mathrm{~nm}$ ) Ag honeycombs electrodes with fine morphology were produced due to the cold machining of the top-flat square ns LDW ablation process in the thin film without heat diffusion. Furthermore, compared to Gaussian beam LDW ablation process, the top-flat square ns LDW ablation efficiently reduces the roughness of the metallic electrode and highly increases the light transmittance to $85.1 \%$. In this condition, the top-flat square LDW ablation processing not only maintains Ag mesh with higher electrical conductivity but also higher transparency. Cyclic bending, folding, twisting, squeezing and ultrasonic bath tests confirm robust adhesion of the Ag honeycomb electrodes on both PEN and quartz substrates. In addition, the transmittance and conductivity of the Ag square honeycombs electrode are high enough to fabricate the transparent touch screen panels without any protective layer over the electrodes.

Funding. Acknowledge the National Natural Science Foundation of China (61705117); Natural Science Foundation of Ningbo (2018A610043); and Chinese Scholars Council. And acknowledge the support of 3315 innovation team, Ningbo city. It was also support by K.C. Wong Magna Fund in Ningbo University.

\section{References}


D. Lee, D. Paeng, H. K. Park, and C. P. Grigoropoulos, "VacuumFree, Maskless Patterning of Ni Electrodes by Laser Reductive Sintering of $\mathrm{NiO}$ Nanoparticle Ink and Its Application to Transparent Conductors," Acs Nano 8, 9807-9814 (2014).

$2 . \quad$ S. Hong, J. Yeo, G. Kim, D. Kim, H. Lee, J. Kwon, H. Lee, P. Lee, and S. H. Ko, "Nonvacuum, Maskless Fabrication of a Flexible Metal Grid Transparent Conductor by Low-Temperature Selective Laser Sintering of Nanoparticle Ink," Acs Nano 7, 50245031 (2013).

3. L. G. De Arco, Y. Zhang, C. W. Schlenker, K. Ryu, M. E. Thompson, and C. W. Zhou, "Continuous, Highly Flexible, and Transparent Graphene Films by Chemical Vapor Deposition for Organic Photovoltaics," Acs Nano 4, 2865-2873 (2010).

4. Y. H. Ho, K. Y. Chen, S. W. Liu, Y. T. Chang, D. W. Huang, and P. K. Wei, "Transparent and conductive metallic electrodes fabricated by using nanosphere lithography," Org Electron 12, 961-965 (2011).

5. Z. B. Yu, Q. W. Zhang, L. Li, Q. Chen, X. F. Niu, J. Liu, and Q. B. Pei, "Highly Flexible Silver Nanowire Electrodes for ShapeMemory Polymer Light-Emitting Diodes," Advanced materials 23, 664-+ (2011).

6. S. R. Ye, A. R. Rathmell, Z. F. Chen, I. E. Stewart, and B. J. Wiley, "Metal Nanowire Networks: The Next Generation of Transparent Conductors," Advanced materials 26, 6670-6687 (2014).

7. W. X. Guo, Z. J. Xu, F. Y. Zhang, S. Y. Xie, H. Y. Xu, and X. Y. Liu, "Recent Development of Transparent Conducting Oxide-Free Flexible Thin-Film Solar Cells," Adv Funct Mater 26, 8855-8884 (2016).

8. O. Inganas, "Organic Photovoltaics Avoiding Indium," Nat Photonics 5, 201-202 (2011).

9. D. S. Hecht, L. B. Hu, and G. Irvin, "Emerging Transparent Electrodes Based on Thin Films of Carbon Nanotubes, Graphene, and Metallic Nanostructures," Advanced materials 23, 1482-1513 (2011).

10. S. Han, S. Hong, J. Ham, J. Yeo, J. Lee, B. Kang, P. Lee, J. Kwon, S. S. Lee, M. Y. Yang, and S. H. Ko, "Fast Plasmonic Laser Nanowelding for a Cu-Nanowire Percolation Network for Flexible Transparent Conductors and Stretchable Electronics," Advanced materials 26, 5808-5814 (2014).

$11 . \quad$ D. H. Zhang, K. Ryu, X. L. Liu, E. Polikarpov, J. Ly, M. E. Tompson, and C. W. Zhou, "Transparent, conductive, and flexible carbon nanotube films and their application in organic light-emitting diodes," Nano Lett 6, 1880-1886 (2006).

12. G. Eda, G. Fanchini, and M. Chhowalla, "Large-area ultrathin films of reduced graphene oxide as a transparent and flexible electronic material," Nat Nanotechnol 3, 270-274 (2008).

13. M. Song, D. S. You, K. Lim, S. Park, S. Jung, C. S. Kim, D. H. Kim, D. G. Kim, J. K. Kim, J. Park, Y. C. Kang, J. Heo, S. H. Jin, J. H. Park, and J. W. Kang, "Highly Efficient and Bendable Organic Solar Cells with Solution-Processed Silver Nanowire Electrodes," Adv Funct Mater 23, 4177-4184 (2013).

14. D. J. Joe, S. Kim, J. H. Park, D. Y. Park, H. E. Lee, T. H. Im, I. Choi, R. S. Ruoff, and K. J. Lee, "Laser-Material Interactions for Flexible Applications," Advanced materials 29(2017).

15. H. B. Lee, W. Y. Jin, M. M. Ovhal, N. Kumar, and J. W. Kang "Flexible transparent conducting electrodes based on metal meshes for organic optoelectronic device applications: a review," J Mater Chem C 7, 1087-1110 (2019).

16. S. R. Ye, A. R. Rathmell, I. E. Stewart, Y. C. Ha, A. R. Wilson, Z. F. Chen, and B. J. Wiley, "A rapid synthesis of high aspect ratio copper nanowires for high-performance transparent conducting films," Chem Commun 50, 2562-2564 (2014).

17. J. Z. Song, J. H. Li, J. Y. Xu, and H. B. Zeng, "Superstable Transparent Conductive Cu@Cu4Ni Nanowire Elastomer Composites against Oxidation, Bending, Stretching, and Twisting for Flexible and Stretchable Optoelectronics," Nano Lett 14, 6298-6305 (2014).
18. S. Hong, H. Lee, J. Yeo, and S. H. Ko, "Digital selective laser methods for nanomaterials: From synthesis to processing," Nano Today 11, 547-564 (2016).

19. V. B. Nam, J. Shin, Y. Yoon, T. T. Giang, J. Kwon, Y. D. Suh, J. Yeo, S. Hong, S. H. Ko, and D. Lee, "Highly Stable Ni-Based Flexible Transparent Conducting Panels Fabricated by Laser Digital Patterning," Adv Funct Mater 29(2019).

$20 . \quad$ D. Paeng, D. Lee, and C. P. Grigoropoulos, "Characteristic time scales of coalescence of silver nanocomposite and nanoparticle films induced by continuous wave laser irradiation," Applied Physics Letters 105(2014)

21. D. Paeng, D. Lee, J. Yeo, J. H. Yoo, F. I. Allen, E. Kim, H. So, H. K. Park, A. M. Minor, and C. P. Grigoropoulos, "Laser-Induced Reductive Sintering of Nickel Oxide Nanoparticles under Ambient Conditions," J Phys Chem C 119, 6363-6372 (2015).

$22 . \quad$ D. Paeng, J. Yeo, D. Lee, S. J. Moon, and C. P. Grigoropoulos, "Laser wavelength effect on laser-induced photo-thermal sintering of silver nanoparticles," Appl Phys a-Mater 120, 12291240 (2015)

23. D. F. Qi, D. Paeng, J. Yeo, E. Kim, L. T. Wang, S. Y. Chen, and C. P. Grigoropoulos, "Time-resolved analysis of thickness-dependent dewetting and ablation of silver films upon nanosecond laser irradiation," Applied Physics Letters 108(2016).

24. D. Paeng, J. H. Yoo, J. Yeo, D. Lee, E. Kim, S. H. Ko, and C. P. Grigoropoulos, "Low-Cost Facile Fabrication of Flexible Transparent Copper Electrodes by Nanosecond Laser Ablation," Advanced materials 27, 2762-+ (2015).

$25 . \quad$ A. D. Wang, L. Jiang, X. W. Li, Y. Liu, X. Z. Dong, L. T. Qu, X. M. Duan, and Y. F. Lu, "Mask-Free Patterning of High-Conductivity Metal Nanowires in Open Air by Spatially Modulated Femtosecond Laser Pulses," Advanced materials 27, 6238-6243 (2015).

26. S. De, P. J. King, P. E. Lyons, U. Khan, and J. N. Coleman, "Size Effects and the Problem with Percolation in Nanostructured Transparent Conductors," Acs Nano 4, 7064-7072 (2010).

27. H. Wu, L. B. Hu, M. W. Rowell, D. S. Kong, J. J. Cha, J. R. McDonough, J. Zhu, Y. A. Yang, M. D. McGehee, and Y. Cui, "Electrospun Metal Nanofiber Webs as High-Performance Transparent Electrode," Nano Lett 10, 4242-4248 (2010). 